( 2021 , The Authors. Published by Elsevier Inc. and Fass Inc. on behalf of the American Dairy Science Association ${ }^{\circledR}$. This is an open access article under the CC BY-NC-ND license (http://creativecommons.org/licenses/by-nc-nd/4.0/).

\title{
Short communication: Comparative performance of 3 on-farm culture systems for detection of mastitis pathogens interpreted by trained and untrained observers
}

\author{
A. Sipka, ${ }^{1 *}$ M. Wieland, ${ }^{1}$ F. Biscarini, ${ }^{2}$ R. M. Rossi ${ }^{1}$ N. Roman, ${ }^{1}$ C. Santisteban, ${ }^{1}$ P. Moroni,,${ }^{1,3}$ and D. V. Nydam ${ }^{1}$ \\ ${ }^{1}$ Department of Population Medicine and Diagnostic Sciences, College of Veterinary Medicine, Cornell University, Ithaca, NY 14853 \\ ${ }^{2}$ Institute of Agricultural Biology and Biotechnology, National Research Council, 20133 Milan, Italy \\ ${ }^{3}$ Università degli Studi di Milano, Dipartimento di Medicina Veterinaria, 26900 Lodi, Italy
}

\begin{abstract}
On-farm culture (OFC) systems facilitate pathogenbased mastitis management and can facilitate antimicrobial stewardship on dairy farms. Interpretation of the results, however, may present a challenge for those with limited microbiology experience. Here, we compared results of 3 OFC systems interpreted by trained and untrained observers against results of a standard laboratory reference method (aerobic culture and mass spectrometry). Milk samples (280 quarter and 60 composite) were selected from submissions for routine diagnostic testing to Quality Milk Production Services (Cornell University, Ithaca, NY) between August 2017 and January 2018. Samples were cultured simultaneously using the standard laboratory reference method and 3 commercially available OFC systems that varied in detail of pathogen identification (provided in parentheses) as follows: (1) Minnesota Easy Culture System II Bi-plate (University of Minnesota Laboratory for Udder Health, St. Paul; gram-positive, gram-negative), (2) Minnesota Easy Culture System II Tri-plate (gram-positive, gram-negative, some genus level), and (3) FERA Diagnostics and Biologicals AccuMast plate (Ithaca, NY; genus level, some species level). After 18 to $24 \mathrm{~h}$ of incubation, OFC plates were interpreted by 1 trained observer ( $>10 \mathrm{yr}$ of experience in milk microbiology) and 6 untrained observers with no previous milk microbiology training, using only the manufacturers' instructions for guidance. Strength of agreement $(\kappa)$ between observer groups and the reference method was determined for the available outcomes of each system. Interpreted by the trained observer, agreement was moderate for identifying gram-positive organisms (Bi-plate, $\kappa=0.56$ ) and substantial for Streptococcus spp. (Tri-plate, $\kappa=0.64$, AccuMast $\kappa$
\end{abstract}

Received June 26, 2020.

Accepted November 19, 2020.

*Corresponding author: ass233@cornell.edu
$=0.61)$. Interpretation by untrained observers resulted in fair agreement $(\kappa=0.29-0.37)$ for these organisms. Moderate agreement $(\kappa=0.43-0.59)$ was found across all 3 OFC for the identification of gram-negative organisms (Bi-plate), non-aureus staphylococci (Tri-plate and AccuMast), Lactococcus spp., and Enterococcus spp. (AccuMast) when interpreted by the trained observer, and fair to moderate agreement was found ( $\kappa$ $=0.31-0.53)$ among untrained observers. Across all 3 OFC, agreement was almost perfect $(\kappa=0.80-0.89)$ for Staphylococcus aureus for the trained observer, and moderate to substantial $(\kappa=0.56-0.61)$ for untrained observers. We concluded that all 3 OFC appeared suitable to support pathogen-based mastitis management when operated by trained observers. Training beyond the instruction manual is a prerequisite to make OFC systems useful for pathogen-based mastitis management.

Key words: mastitis, on-farm culture, test evaluation, training

\section{Short Communication}

On-farm culture (OFC) systems are widely used to facilitate pathogen-based mastitis management on dairy farms (Lago and Godden, 2018). Their implementation has been shown to reduce antimicrobial usage without adversely affecting cow health and performance (Vasquez et al., 2017; McDougall et al., 2018). Among commercially available products, the Minnesota Easy Culture System II Bi-plate and Tri-plate (University of Minnesota Laboratory for Udder Health, St. Paul;), and the AccuMast plate by FERA Diagnostics and Biologicals (Ithaca, NY) represent OFC using selective (Bi- and Tri-plate) and chromogenic (AccuMast) media-detection mechanisms (Ganda et al., 2016; Lago and Godden, 2018). The systems vary in detail of pathogen identification from broad distinction between gram-positive and gram-negative organisms (Bi-plate) to genus specific (Tri-plate) and species level identifica- 
tion for some organisms (AccuMast). A detailed description of the diagnostic capacities for the 3 different OFC systems is provided in Supplemental File S1 and Supplemental Table S1 (https://figshare.com/articles/ dataset/Supplemental_tables_S1-S4_docx/13703413). Despite the differences in identification detail, the 3 systems have one thing in common: they all share the need of correct interpretation of the OFC results to allow appropriate treatment decision making (PinzónSánchez et al., 2011). The primary objective of our study was to compare the performance of these 3 commonly used OFC systems with the standard laboratory procedures of aerobic culture followed by MALDI-TOF analysis of bacterial growth. Because few studies have included observers with limited microbiology experience (Royster et al., 2014; Griffioen et al., 2018), a secondary objective was to describe the influence of microbiology experience on the interpretation of $\mathrm{OFC}$ results. This group was intended to represent an average farm employee who would be reading plates in a typical on-farm situation.

Between August 2017 and January 2018, 340 milk samples (280 quarter samples, 60 composite samples) were selected from routine submissions to Quality Milk Production Services (Cornell University, Ithaca, NY) by commercial dairy farms for mastitis pathogen identification services. Samples were selected after aerobic culture and MALDI-TOF identification and stored at $-20^{\circ} \mathrm{C}$ until used for the study. We attempted to represent a sufficient number and variety of commonly observed culture outcomes and selected samples identified with major and minor mastitis pathogens, contaminated samples, and samples with no bacterial growth. When samples were cultured on the OFC systems, the reference method was repeated, and OFC results were compared with the second time the reference method was performed to best represent contemporaneous comparison to the reference test.

An experienced laboratory technician, who was not assigned to read out plates, performed all aerobic bacteriologic inoculation of the milk samples according to the Laboratory Handbook on Bovine Mastitis (NMC, 2017). Briefly, samples were thawed, mixed, and plated on the respective culture medium using a sterile cotton swab (Puritan Medical Products Co., Guilford, ME; cotton swab dimensions, $16 \times 5 \mathrm{~mm}$ ), which resulted in approximately $30 \mu \mathrm{L}$ of milk per medium section. One sheep blood agar plate (Northeast Laboratory, Waterville, ME) per sample was used for the reference method of aerobic culture with subsequent MALDI-TOF identification. For the OFC systems, 1 cotton swab per medium section was used to inoculate the sample. All plates were incubated at $37^{\circ} \mathrm{C}$. On-farm culture plates were read after 18 to $24 \mathrm{~h}$; blood agar plates for the ref- erence method were incubated and were read after 18 to $24 \mathrm{~h}$ and again after $48 \mathrm{~h}$. Samples were cultured in sets of 20 per session. The OFC plates were numbered 1 to 20 such that the number assigned to each sample was different on each OFC system to avoid comparison between the 3 systems by the observers. That is, sample 1 on the Bi-plate did not correspond to sample 1 on the Tri-plate or AccuMast plate in an attempt to control bias during each diagnostic session and have independence in diagnosis among all 3 OFC systems.

For the reference method, culture results were interpreted following Quality Milk Production Services standard protocols adapted from Dohoo et al. (2011). Cultures were considered positive if there was $\geq 1 \mathrm{cfu}$ of Staphylococcus aureus or Streptococcus agalactiae, $\geq 6$ cfu of NAS, or $\geq 3$ cfu for all other organisms. Subsequently, a representative colony was submitted for MALDI-TOF analysis using the MALDI Biotyper Microflex LT (Bruker Daltonics, Billerica, MA). Adaption and maintenance of the MALDI-TOF library, sample preparation, and interpretation of the MALDI score were performed as described by Randall et al. (2015). In brief, the instrument reports a logarithmic score between 0 and 3 , quantifying similarity to known database entries. A log (score) $\geq 1.7$ was the threshold for the genus level identification, and a $\log$ (score) $\geq 2.0$ was the set threshold for a match at the species level. If there were $\geq 3$ phenotypically different organisms present, the sample was considered contaminated, and no MALDI-TOF analysis was performed.

The OFC plates were interpreted by 1 trained milk microbiologist and 6 veterinary students with no previous specific milk microbiology training who represented untrained observers, as would be typical of on-farm employees. To facilitate consistent focus and prevent mental fatigue of observers, we allocated the samples into 17 sessions. Untrained observers were provided with the manufacturer's instructions for the respective system (MN Easy Culture System User's Guide 2016 (https://www.vdl.umn.edu/sites/vdl.umn.edu/files/ mn-easy-culture-system-ii-users-guide-english.pdf) and AccuMast culture identification chart). Observers were masked to each other's results, as well as to the result of the reference method.

Outcomes were evaluated based on the diagnostic capacity of the respective OFC system (Supplemental Table S1). Observers were expected to identify mixed growth according to the capacity of the respective OFC. The identification of Staph. aureus was prioritized in mixed samples, and observers were expected to identify Staph. aureus among 2 organisms for all 3 OFC systems.

The trained observer conducted all sessions, whereas attendance of untrained observers varied. To compile a 
complete set of observations from untrained observers and give each observation equal weight in the analyses, we randomly selected results from 1 untrained observer per session using an open source random number generator (Haahr, 2020) in an attempt to simulate an average on-farm employee and maintain independence of each analysis. There was no comparison among the untrained observers, and each untrained observer was independent and identical to the others. Data were maintained in spreadsheet software (Microsoft Office Excel 2016; Microsoft Corporation, Redmond, WA). Before statistical analyses, we screened the data for missing and erroneous values. Statistical analyses were performed in JMP Pro 14 (SAS Institute Inc., Cary, NC). Sensitivity (Se), specificity (Sp), and positive and negative predictive values (PPV and NPV, respectively) were calculated according to Dohoo et al. (2009) for all available outcomes of the respective OFC and grouped by trained and untrained observers, using MALDI-TOF results as the reference method. The $\kappa$ statistics were calculated to measure agreement beyond chance between observer groups and the reference method. We interpreted $\kappa$ values according to the guideline established by Landis and Koch (1977). A value of $>0.80$ was considered as almost perfect, $>0.60$ to 0.80 as substantial, $>0.40$ to 0.60 as moderate, $>0.20$ to 0.40 as fair, $>0.00$ to 0.20 as slight agreement, and $\leq 0.00$ as poor agreement. For Se, Sp, PPV, and NPV, values $>0.80$ were interpreted as high, $>0.60$ as intermediate, and $\leq 0.60$ as low according to Royster et al. (2014). The $95 \%$ confidence intervals for Se, Sp, PPV, $\mathrm{NPV}$, and $\kappa$ values were calculated as $a \pm 1.96 \times$ standard error (SE) where $\mathrm{SE}=\sqrt{a(1-a) / n}$, with $a=$ the test proportion or $\kappa$ value and $n=$ the sample size.

A total of 340 samples interpreted by the trained observer and untrained observers on all 3 OFC in 17 sessions were available for analysis. Following random selection, 3 untrained observers contributed 2 sessions each, and 1 untrained observer each contributed 1, 4, or 6 sessions, respectively, to the final data set. Thirteen observations were missing, resulting in a total of 2,027 records across all 3 OFC available for final analyses. The frequency distribution of culture outcomes determined by the reference method for the individual samples is summarized in Table 1. Table 2 summarizes $\kappa$ statistics stratified by OFC system and observer group. Values for Se, Sp, PPV, and NPV are summarized in Supplemental Tables S2 through S4, respectively.

Interpreted by the trained observer, the "grampositive" outcome on the Bi-plate system, the genus specific gram-positive outcomes "Streptococcus spp. or Streptococcus-like organisms (SLO)" on the Tri-plate system, and outcome "Streptococcus spp." on the Ac-
cuMast system showed substantial agreement and intermediate Se $(0.76,0.67$, and 0.77 , respectively). Streptococcus spp. and SLO are the most frequently treated mastitis pathogens (Neeser et al., 2006). Based on previous reports (Royster et al., 2014; Lago and Godden, 2018), we expected better test statistics with less complex OFC systems. However, our data suggested that all 3 OFC were equally suitable to support treatment protocols based on the detection of gram-positive organisms or Streptococcus spp. if results were interpreted by a trained observer. Detection of Enterococcus spp. and Lactococcus spp. (EL) was only possible with the AccuMast system and had a low Se for the trained (0.34) and untrained (0.47) observers in this study. Ferreira and colleagues (2018) reported a higher Se (0.95) for the outcome EL. Our results suggested that an auxiliary laboratory, using either biochemistry or MALDI-TOF, might be necessary to provide support for the reliable detection of these pathogens. We found that untrained observers had only fair agreement and low Se (0.58) for the outcome "gram-positive" in the Bi-plate system compared with the trained observer. Identification of gram-positive pathogens on genus level using the Tri-plate and AccuMast system was simi-

Table 1. Frequency distribution of culture outcomes from 340 prospectively selected milk samples using aerobic culture and MALDITOF

\begin{tabular}{|c|c|c|}
\hline Culture result & Number & Percent $^{1}$ \\
\hline No growth & 77 & 22.6 \\
\hline Gram-positive & 172 & 50.5 \\
\hline Staphylococcus aureus & 32 & 9.4 \\
\hline Staphylococcus spp. & 31 & 9.1 \\
\hline Streptococcus dysgalactiae & 29 & 8.5 \\
\hline Streptococcus uberis & 18 & 5.3 \\
\hline Streptococcus spp. & 10 & 2.9 \\
\hline Streptococcus agalactiae & 4 & 1.2 \\
\hline Lactococcus spp. & 24 & 7.1 \\
\hline Enterococcus spp. & 8 & 2.4 \\
\hline Trueperella pyogenes & 7 & 2.1 \\
\hline Gram-positive bacillus & 9 & 2.6 \\
\hline Gram-negative & 39 & 11.5 \\
\hline Escherichia coli & 18 & 5.3 \\
\hline Klebsiella spp. & 12 & 3.5 \\
\hline Enterobacter spp. & 3 & 1.0 \\
\hline Citrobacter spp. & 1 & 0.3 \\
\hline Serratia spp. & 3 & 1.0 \\
\hline Gram-negative bacillus & 2 & 0.6 \\
\hline Mixed $^{2}$ & 20 & 5.9 \\
\hline Other & 18 & 5.3 \\
\hline Yeast & 10 & 2.9 \\
\hline Prototheca spp. & 6 & 1.8 \\
\hline Mold & 2 & 0.6 \\
\hline Contamination $^{3}$ & 14 & 4.1 \\
\hline
\end{tabular}

${ }^{1}$ Percentages do not add up to $100 \%$ because of rounding error.

${ }^{2}$ Sample grew 2 distinct organisms, neither of them Staph. aureus or Strep. agalactiae.

${ }^{3}$ Sample grew 3 or more different organisms and no contagious pathogen such as Staph. aureus or Strep. agalactiae were present. 
Table 2. Strength of agreement beyond chance ( $\kappa$ statistics) with the reference method of aerobic culture and MALDI-TOF for 3 different onfarm culture systems

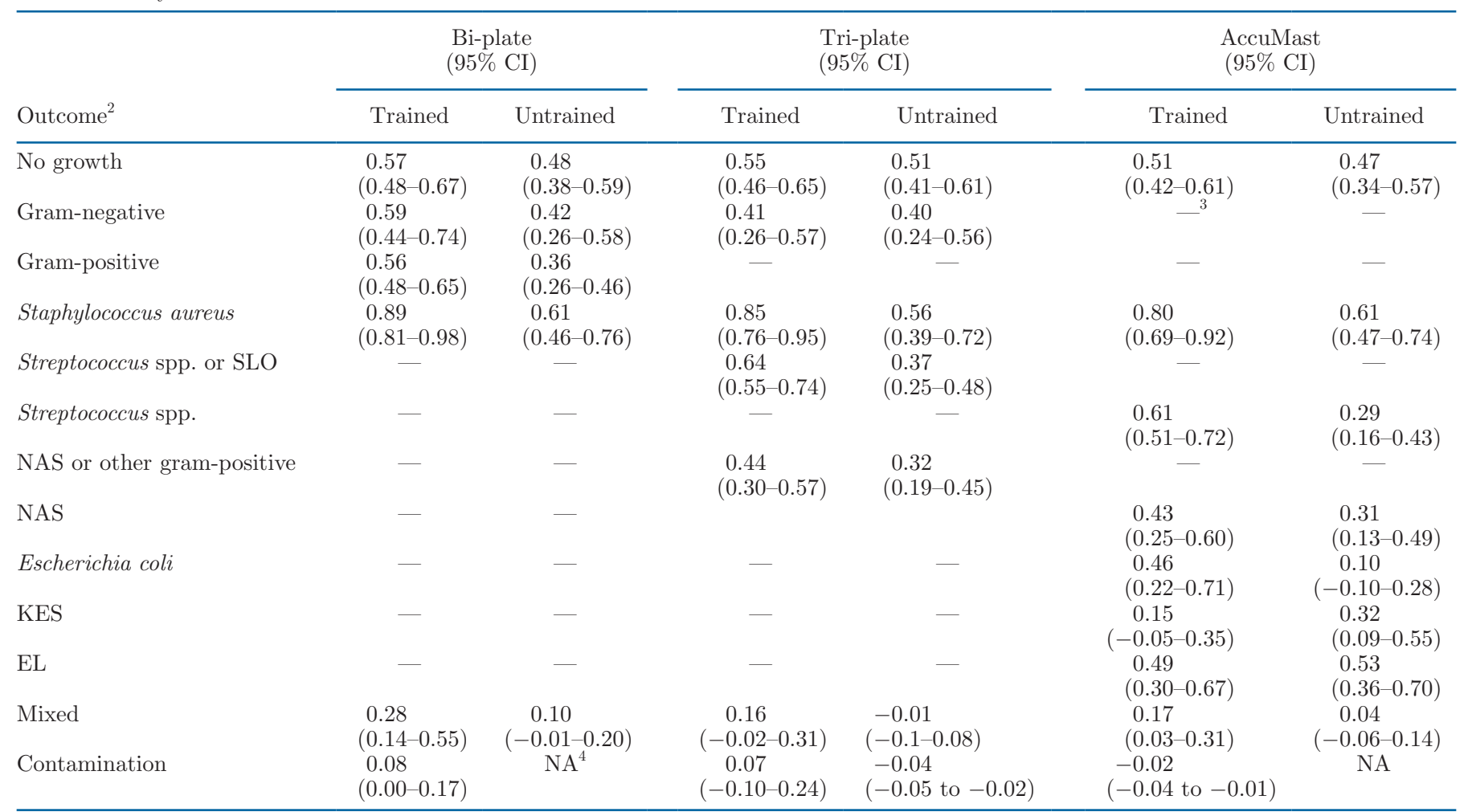

${ }^{1}$ On-farm culture systems: Minnesota Easy Culture Bi-plate and Tri-plate systems (University of Minnesota Laboratory for Udder Health, St. Paul, MN) and the AccuMast system (FERA Diagnostics and Biologicals, Ithaca, NY)]; the $\kappa$ values and 95\% CI were calculated for all interpretable outcomes of each system and stratified by trained and untrained observers, respectively.

${ }^{2} \mathrm{SLO}=$ Streptococcus-like organisms including Enterococcus spp. and Lactococcus spp.; other gram-positive = gram-positive bacillus and Trueperella pyogenes; KES = Klebsiella spp., Enterobacter spp., Serratia spp.; EL = Enterococcus spp. or Lactococcus spp.; mixed = sample grew 2 distinct organisms, neither of them Staph. aureus or Streptococcus agalactiae; contamination = sample grew 3 or more different organisms and no contagious pathogen such as Staph. aureus or Strep. agalactiae were present.

$3_{-}=$outcome not available on respective OFC.

${ }^{4} \mathrm{NA}=$ not applicable. Outcome was not identified by untrained observers.

larly challenging for untrained observers. Strength of agreement was fair and Se was low $(<0.50)$ in the Triplate system for Streptococcus spp. or SLO, and in the AccuMast system for Streptococcus spp. and EL. For NAS, both trained and untrained observers showed moderate to fair strength of agreement, and comparable, low Se $(\leq 0.55)$ and PPV $(\leq 0.52)$ on Tri-plate and AccuMast system.

Interpreted by the trained observer, all 3 OFC systems performed comparably well in detecting Staph. aureus, with almost perfect (Bi- and Tri-plate system) to substantial agreement and overall high Se $(>0.80$ for $\mathrm{Bi}$ - and Tri-plate) to intermediate $\mathrm{Se}(0.78$ for AccuMast). These findings are consistent with previous studies that show substantial agreement and high Se for the Bi-and Tri-plate system (McCarron et al., 2009; Royster et al., 2014). For the AccuMast system, Ganda and colleagues (2016) reported a higher Se (1.00), albeit based on a very low number of Staph. aureus positive samples $(\mathrm{n}=2)$. Specificity and PPV for Staph. aureus were high for all 3 OFC $(\mathrm{Sp}=0.99$, PPV $=0.80-0.93)$ for the trained observer. Considering the consequences of false-positive results (e.g., segregation or culling of a diagnosed cow), it is advisable to support results that are positive or suspect for Staph. aureus with a confirmatory test (Lago and Godden, 2018). The detection of Staph. aureus on the Bi- and Tri-plates was more difficult for untrained observers, as shown by lower Se (0.63 and 0.47 respectively) and lower $\kappa$ values compared with the trained observer. The colorimetric approach of the AccuMast system appeared to be better for detection of samples positive for Staph. aureus for untrained observers (Se 0.80). The untrained observers' Sp and PPV for this outcome, however, were lower (0.93 and 0.55 respectively) for the AccuMast system, and the Bi-and Tri-plates had higher Sp $(>0.95)$ and PPV $(>0.65)$. Our results showed that relying solely on the manufacturer's instructions potentially increases 
the risk of both false-negative and false-positive results. This can lead to an increased risk of transmission (in the case of false-negative results) or needless segregation, treatment, or removal from the herd (in the case of false-positive results).

Detection of gram-negative pathogens showed overall low Se $(\leq 0.50)$ for both observer groups. Strength of agreement was moderate to fair for the $\mathrm{Bi}$ - and Triplate system, and moderate to slight for the AccuMast system. The benefits of treating mastitis caused by gram-negative organisms are controversially discussed (Schukken et al., 2011; Fuenzalida and Ruegg, 2019). A common management strategy is to only treat severe clinical cases (Constable et al., 2009; Lago et al., 2011). Failure to identify gram-negative growth due to a misclassification of "no growth" would not lead to a different decision for this management strategy. However, among untrained observers, growth of gram-negative organisms was frequently misclassified as growth in all parts of the OFC plates, especially for the AccuMast system. This could result in an increase of intramammary antimicrobial use, consequently increasing treatment costs, risk of bulk tank antibiotic residue violations, and possibly the transfer of antibiotic resistant bacteria to the food supply. Griffioen and colleagues (2018) found similar results for OFC systems using other chromogenic media.

Both observer groups performed comparably in identifying the outcome "no growth" across all 3 OFC, showing moderate agreement for all 3 OFC systems and low PPV. A similarly high rate of false-positive results for this outcome was shown by others (Royster et al., 2014; Ferreira et al., 2018). From a practical point of view, this would mean that the farm management could miss cows that should have been treated.

Mixed growth was hard to correctly identify across all 3 OFC for both trained and untrained observers, with only fair to poor agreement and low Se $(<0.60)$. In scoring mixed growth, we strictly adhered to the respective OFC system's capacity, which means we expected observers to distinguish between Streptococcus spp. or SLO and growth of other gram-positive organisms for the Tri-plate system, and between the various options for gram-negative and gram-positive pathogens groups in the AccuMast system. As a result, the Biplate system showed the best test statistics for this category, considering that the only detectable mixed growth was a mix of gram-negative and gram-positive growth. Royster and colleagues (2014) found similar Se and $\mathrm{Sp}$ for mixed growth in the Bi- and Tri-plate systems, albeit paired with a higher PPV. This is mostly due to a higher prevalence of mixed samples in their study (>20\%) compared with our study $(5.9 \%)$.
The outcome "contamination" had only slight to poor agreement across all 3 OFC. Untrained observers did not interpret any growth as "contaminated" using the Bi-plate or the AccuMast system, although this culture outcome is described in the manual of the Minnesota Easy Culture System. Difficulties to recognize contaminated cultures were also described by others (Royster et al., 2014; Griffioen et al., 2018). Together, these results emphasized the importance of proper sampling procedures to minimize the frequency of contaminated samples, as well as the need for additional targeted training for this specific culture result.

A limitation of our study was the selection of samples based on their results from prior culture and MALDITOF and freezing them until they were used for the study. We intended this to be an advantage of our study so that we could have a representative selection of diagnostic results for mastitis as well as contemporaneously evaluate the reference and test methods. However, as reported by Schukken and colleagues (1989), freezing can lead to a decrease of colony-forming units in gramnegative organisms, which helps explain why not all gram-negative organisms could be recovered from the enrolled samples when cultured for our study. This increased the number of samples with "no growth"; however, with an overall prevalence of $22.6 \%$, our study is still within the range of prevalence for no bacteriologic growth reported by others (Lago and Godden, 2018).

Our data show that experience in milk microbiology substantially improved interpretation of OFC culture results, indicating that specific, hands-on training beyond the use of the manufacturer's instruction is crucial to facilitate appropriate management decisions. When interpreted by a trained observer, all 3 tested OFC systems showed comparable performance and appeared suitable to support common mastitis treatment protocols. Our results further suggest that for certain organisms, confirmation (e.g., Staph. aureus) or supportive diagnostics (e.g., EL, gram-negative organisms) by an auxiliary laboratory service is recommended.

\section{ACKNOWLEDGMENTS}

This project was funded in part by the Residents Research Grants Program of the College for Veterinary Medicine, Cornell University (Ithaca, NY). The authors acknowledge the skillful assistance of Rachel Slater and Jeff Runyan (both from Cornell University, Ithaca, NY). F. Biscarini is currently seconded at the European Research Council Executive Agency (ERCEA; Brussels, Belgium); the views expressed here are purely those of the writer and may not in any circumstances be regarded as stating an official position of the 
European Commission. The authors have no conflict of interest.

\section{REFERENCES}

Constable, P. D., S. Pyorala, and G. W. Smith. 2009. Guidelines for Antimicrobial Use in Cattle. Pages $143-160$ in Guide to Antimicrobial Use in Animals. L. Guardabassi, L. B. Jensen, and H. Kruse, ed. Blackwell Publishing Ltd., Oxford, UK.

Dohoo, I., W. Martin, and H. Stryhn. 2009. Screening and diagnostic tests. Pages 91-134 in Veterinary Epidemiologic Research. 2nd ed. AVC Inc., Charlottetown, Prince Edward Island, Canada.

Dohoo, I. R., J. Smith, S. Andersen, D. F. Kelton, and S. Godden. 2011. Diagnosing intramammary infections: Evaluation of definitions based on a single milk sample. J. Dairy Sci. 94:250-261. https://doi.org/10.3168/jds.2010-3559.

Ferreira, J. C., M. S. Gomes, E. C. R. Bonsaglia, I. F. Canisso, E. F. Garrett, J. L. Stewart, Z. Zhou, and F. S. Lima. 2018. Comparative analysis of four commercial on-farm culture methods to identify bacteria associated with clinical mastitis in dairy cattle. PLoS One 13:e0194211. https://doi.org/10.1371/journal.pone.0194211.

Fuenzalida, M. J., and P. L. Ruegg. 2019. Negatively controlled, randomized clinical trial to evaluate intramammary treatment of nonsevere, gram-negative clinical mastitis. J. Dairy Sci. 102:5438 5457. https://doi.org/10.3168/jds.2018-16156.

Ganda, E. K., R. S. Bisinotto, D. H. Decter, and R. C. Bicalho. 2016. Evaluation of an on-farm culture system (Accumast) for fast identification of milk pathogens associated with clinical mastitis in dairy cows. PLoS One 11:e0155314. https://doi.org/10.1371/ journal.pone. 0155314 .

Griffioen, K., A. G. J. Velthuis, L. A. Lagerwerf, A. E. Heuvelink, and T. Lam. 2018. Agreement between four commercial diagnostic tests and routine bacteriological culture of milk to determine the udder infection status of dairy cows. Prev. Vet. Med. 157:162-173. https://doi.org/10.1016/j.prevetmed.2018.07.003.

Haahr, M. 2020. RANDOM.ORG: True Random Number Service. Randomness and Integrity Services Ltd. Accessed Sep. 24, 2020. https://www.random.org/.

Lago, A., and S. M. Godden. 2018. Use of rapid culture systems to guide clinical mastitis treatment decisions. Vet. Clin. North Am. Food Anim. Pract. 34:389-412. https://doi.org/10.1016/j.cvfa .2018.06.001.

Lago, A., S. M. Godden, R. Bey, P. L. Ruegg, and K. Leslie. 2011. The selective treatment of clinical mastitis based on on-farm culture results: II. Effects on lactation performance, including clinical mastitis recurrence, somatic cell count, milk production, and cow survival. J. Dairy Sci. 94:4457-4467. https://doi.org/10.3168/jds $.2010-4047$.
Landis, J. R., and G. G. Koch. 1977. The measurement of observer agreement for categorical data. Biometrics 33:159-174. https://doi .org $/ 10.2307 / 2529310$.

McCarron, J. L., G. P. Keefe, S. L. McKenna, I. R. Dohoo, and D. E. Poole. 2009. Laboratory evaluation of 3M Petrifilms and University of Minnesota Bi-plates as potential on-farm tests for clinical mastitis. J. Dairy Sci. 92:2297-2305. https://doi.org/10.3168/jds .2008-1661.

McDougall, S., J. Niethammer, and E. M. Graham. 2018. Antimicrobial usage and risk of retreatment for mild to moderate clinical mastitis cases on dairy farms following on-farm bacterial culture and selective therapy. N. Z. Vet. J. 66:98-107. https://doi.org/10 .1080/00480169.2017.1416692.

Neeser, N. L., W. D. Hueston, S. M. Godden, and R. F. Bey. 2006. Evaluation of the use of an on-farm system for bacteriologic culture of milk from cows with low-grade mastitis. J. Am. Vet. Med. Assoc. 228:254-260. https://doi.org/10.2460/javma.228.2.254.

NMC. 2017. Laboratory Handbook on Bovine Mastitis. National Mastitis Council, Madison, WI.

Pinzón-Sánchez, C., V. E. Cabrera, and P. L. Ruegg. 2011. Decision tree analysis of treatment strategies for mild and moderate cases of clinical mastitis occurring in early lactation. J. Dairy Sci. 94:18731892. https://doi.org/10.3168/jds.2010-3930.

Randall, L. P., F. Lemma, M. Koylass, J. Rogers, R. D. Ayling, D. Worth, M. Klita, A. Steventon, K. Line, P. Wragg, J. Muchowski, M. Kostrzewa, and A. M. Whatmore. 2015. Evaluation of MALDI$\mathrm{ToF}$ as a method for the identification of bacteria in the veterinary diagnostic laboratory. Res. Vet. Sci. 101:42-49. https://doi.org/10 .1016/j.rvsc.2015.05.018.

Royster, E., S. Godden, D. Goulart, A. Dahlke, P. Rapnicki, and J. Timmerman. 2014. Evaluation of the Minnesota Easy Culture System II Bi-Plate and Tri-Plate for identification of common mastitis pathogens in milk. J. Dairy Sci. 97:3648-3659. https://doi.org/ 10.3168/jds.2013-7748.

Schukken, Y. H., G. J. Bennett, M. J. Zurakowski, H. L. Sharkey, B. J. Rauch, M. J. Thomas, B. Ceglowski, R. L. Saltman, N. Belomestnykh, and R. N. Zadoks. 2011. Randomized clinical trial to evaluate the efficacy of a 5-day ceftiofur hydrochloride intramammary treatment on nonsevere gram-negative clinical mastitis. J. Dairy Sci. 94:6203-6215. https://doi.org/10.3168/jds.2011-4290.

Schukken, Y. H., J. A. H. Smit, F. J. Grommers, D. Vandegeer, and A. Brand. 1989. Effect of freezing on bacteriologic culturing of mastitis milk samples. J. Dairy Sci. 72:1900-1906. https://doi.org/ 10.3168/jds.S0022-0302(89)79309-7.

Vasquez, A. K., D. V. Nydam, M. B. Capel, S. Eicker, and P. D. Virkler. 2017. Clinical outcome comparison of immediate blanket treatment versus a delayed pathogen-based treatment protocol for clinical mastitis in a New York dairy herd. J. Dairy Sci. 100:29923003. https://doi.org/10.3168/jds.2016-11614. 